\title{
Performance Evaluation of Radio Frequency Plasma Cathodes for Hall Thrusters
}

\author{
By Hiroki Watanabe, Takanori Deguchi, Shuka TAKedA, Yuki MiURA, Masanori Ichimura and Haruki TAKegahara
}

Tokyo Metropolitan University, Tokyo, Japan

(Received July 27th, 2015)

\begin{abstract}
To liberate Hall thrusters from the drawbacks associated with dispenser hollow cathodes, we construct and experimentally evaluate an outer-coil-type radio frequency (RF) plasma cathode and an inner-coil-type RF plasma cathode. The influence of the coil configuration on the electron-emission characteristics of the RF plasma cathodes is significant. Compared to the inner-coil-type RF plasma cathode, the outer-coil-type RF plasma cathode achieved higher electronemission performance. For the outer-coil-type RF plasma cathode, we obtained an anode current of $3.3 \mathrm{~A}$ at an RF power of $140 \mathrm{~W}$, a xenon mass flow rate of $0.3 \mathrm{mg} / \mathrm{s}$, and an anode voltage of $58 \mathrm{~V}$. The anode current is sufficiently high to operate a 1-kW class Hall thruster. The gas utilization factor for the outer-coil-type RF plasma cathode is comparable to that for a conventional dispenser hollow cathode. On the other hand, the electron production cost for the outer-coil-type RF plasma cathode is four times higher than that for the hollow cathode. Thus, there is a need to improve the power consumption for application of RF plasma cathodes to Hall thrusters.
\end{abstract}

Key Words: Electric Propulsion, Hall Thruster, Cathode, Radio Frequency Discharge

\section{Nomenclature}

$\begin{array}{cll}C_{e} & : \text { electron production cost } \\ I_{a} & : \text { anode current } \\ I_{c} & : \text { cathode current } \\ I_{k} & : \text { keeper current } \\ \dot{m}_{c} & : \text { cathode mass flow rate } \\ P_{r f} & : \text { radio frequency }(\mathrm{RF}) \text { power } \\ U_{e} & : \text { gas utilization factor } \\ V_{a} & : \text { anode voltage } \\ V_{k} & : \text { keeper voltage }\end{array}$

\section{Introduction}

Hall thrusters are frequently used worldwide to realize the orbit control of geostationary satellites, as well as for the orbital transfer of space probes, because they contribute significantly to the shortening of mission trip time and increased the payload ratio ${ }^{1)}$. However, the thrust generated in the thrusters is very small compared to that of conventional chemical rockets. Accordingly, to take advantage of their high specific impulse and high efficiency, long and continuous operation lasting several years is required ${ }^{2}$. Hollow cathodes are regularly employed as electron sources in Hall thrusters to ionize the propellant using electric discharges and to neutralize the ion beam leaving the thrusters ${ }^{3-5)}$. Conventional hollow cathodes use a porous tungsten insert that is impregnated with an emissive mix of barium and calcium oxides, and alumina for thermionic emission ${ }^{6)}$. Chemical reactions between the hot tungsten and the oxides at high temperature lead to the production of free barium, which flows through the pores, and which eventually reaches the tungsten surface. The surface then becomes covered with a monolayer of barium and the thermionic emission increases greatly owing to the very marked reduction in the work function from $4.5 \mathrm{eV}$ to $1.5 \mathrm{eV}^{7}$. Because the dipole effect of this monolayer will be modified by the adsorption of gas or metal vapor onto its surface, particularly if the gas or metal is electro-negative, the work function will increase and cause a decrease in the electron emission, usually termed emission poisoning ${ }^{8)}$. To prevent this poisoning, which can shorten the lifetime of the dispenser hollow cathode, contact between the insert and the active gas should be avoided, and the type of operation gas entering the cathode, which is commonly shared with the thruster propellant, is restricted. In addition, the lifetime of the dispenser hollow cathode is considered to be restricted by oxide depletion from the insert. As a result, it is difficult for Hall thrusters with dispenser hollow cathodes to realize long-term operation, and they should be controlled strictly from prelaunch to end-of-life.

In light of these constraints, the lifetime and erosion mechanisms in dispenser hollow cathodes have been intensively investigated by researchers ${ }^{9-13)}$. First, two solutions have been studied to liberate Hall thrusters from the drawbacks of the dispenser hollow cathode. One is the employment of boride, e.g., lanthanum hexaboride $\left(\mathrm{LaB}_{6}\right)$, as the thermionic emitter in the hollow cathode to replace the barium-impregnated tungsten ${ }^{3,14,15)}$. The other is the use of a "plasma cathode" as the electron source in Hall thrusters. The plasma cathode is an electrical discharge device producing plasma that emits electrons from its boundary ${ }^{16)}$. The $\mathrm{LaB}_{6}$ hollow cathode has a higher resistance to poisoning than the dispenser cathode, and does not require the strict controls that are normally required for the dispenser cathode. However, it requires high-temperature operation $\left(>1,600{ }^{\circ} \mathrm{C}\right)$ to achieve a high emission current density. Thus, the reliability of the heater system is critical issue for the cathode life and the evaporation of $\mathrm{LaB}_{6}$ at high temperatures limits the cathode 
life. Although a plasma cathode requires additional discharge power to produce electrons, it does not have the drawbacks associated with a thermionic emitter, and may achieve high robustness and a long lifetime. Electron cyclotron resonance (ECR) plasma ${ }^{17-19)}$, capacitively coupled plasma $(\mathrm{CCP})^{20)}$, inductively coupled plasma (ICP $)^{21-23)}$, and helicon wave plasma $^{24)}$ have been investigated for cathode applications in electric propulsion. The microwave discharge ion thruster " $\mu 10$ ", which was installed in the Hayabusa asteroid explorer, is an attractive thruster that employs a thermionic-emitter-less design; it employed a microwave ECR discharge to ionize the propellant and a microwave ECR plasma cathode to neutralize the extracted ions. Its accumulated operational time was $35,000 \mathrm{~h}$, and the thruster demonstrated that the removal of the hollow cathode benefits the operation sequence and lifetime of electric propulsion ${ }^{25)}$.

While plasma cathodes are a viable solution, there are challenging problems such as the need to improve the ratio of the electron emission current to the power consumption such that they are comparable to conventional hollow cathodes. The key to obtaining a high electron emission current from plasma cathodes is the production of dense plasma within the cathode. ICP provides a sufficiently high electron number density without the need for an external magnetic field because the plasma does not depend upon a high voltage to drive the displacement current through the powered radio frequency (RF) sheath ${ }^{26)}$. Moreover, the limited electron density, which is attributed to the cutoff frequency, as in ECR plasma, is not important for ICP. Because of these features of ICP, in this study, we focused on a plasma cathode employing a RF discharge as a simple and robust electron source for Hall thrusters. Then we experimentally evaluated the electronemission characteristics of the RF plasma cathode.

\section{Experimental Apparatus and Procedure}

\subsection{Radio frequency plasma cathode}

In this study, we designed and fabricated two RF plasma cathodes. One is an outer-coil-type RF plasma cathode, while the other is an inner-coil-type RF plasma cathode. Figure 1 shows a schematic representation of the outer-coil-type RF plasma cathode, which consists of a discharge vessel, an orifice plate, an induction coil, and an ion collector. The cylindrical vessel is made of alumina, and its inner diameter and length are $40 \mathrm{~mm}$ and $80 \mathrm{~mm}$, respectively. Copper wire whose diameter is $5 \mathrm{~mm}$ is wrapped around the vessel as the induction coil. The orifice plate is made of graphite, and there is a 2-mm diameter orifice at the center of the plate for electron emission. Because an ignition of ICP will obey Paschen's law, the orifice diameter is much smaller than the vessel's inner diameter, and this will enable it to sustain a sufficiently high pressure in the RF cathode for the selfignition of plasma. When electrons are emitted from the orifice, an equivalent quantity of ions then has to be collected at the ion collector in the cathode to maintain quasi-neutrality in the RF plasma. Thus, the graphite collector, which is inserted into the vessel, is necessary during the steady operation of the cathode. The cylindrical collector is inserted

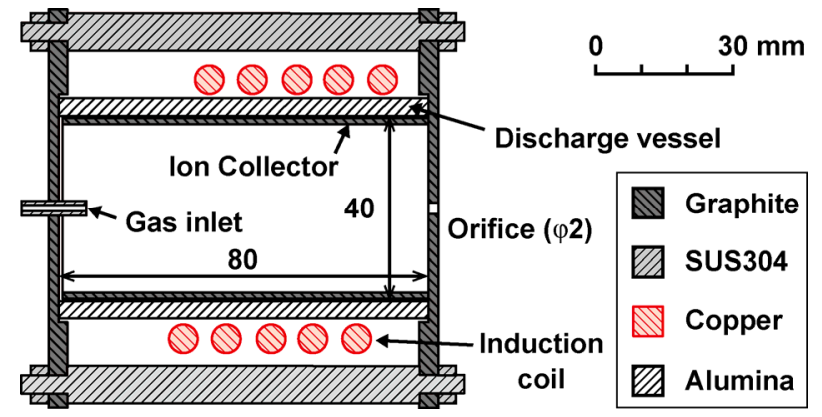

Fig. 1. Cross section of the outer-coil-type RF plasma cathode.

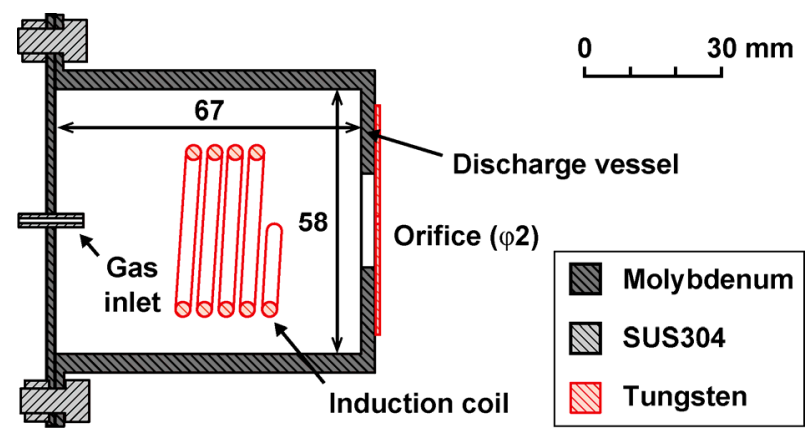

Fig. 2. Cross section of the inner-coil-type RF plasma cathode.

into the vessel along its wall to act as an ion collector and a Faraday shield. The collector has an axial slit that allows the axial $d B / d t$ fields into the plasma, but suppresses the circumferential $d E / d t$ field, effectively serving as a Faraday shield $^{27)}$.

Figure 2 shows a schematic representation of the inner-coiltype RF plasma cathode, which consists of a discharge vessel, an orifice plate, and an induction coil. The cylindrical vessel is made of molybdenum, and its inner diameter and length are 58 $\mathrm{mm}$ and $67 \mathrm{~mm}$, respectively. The orifice plate is made of tungsten. As in the case of the outer-coil-type cathode, there is a 2-mm diameter orifice at the center of the plate for electron emission. The induction coil was made of tungsten wire having a diameter of $3 \mathrm{~mm}$, and was inserted into the vessel. The metal vessel performs the role of the ion collector to maintain quasi-neutrality in the RF plasma during the steady operation of the cathode. Thus, the inner-coil-type cathode does not require an additional ion collector.

\subsection{Experimental setup}

Figure 3 shows the experimental setup. All of the experiments were conducted in a vacuum chamber, whose diameter and length were $1.6 \mathrm{~m}$ and $3.2 \mathrm{~m}$, respectively. The backpressure in the chamber was brought to approximately 6.3 $\times 10^{-4} \mathrm{~Pa}$ by two cryogenic pumps under a xenon mass flow rate of $0.4 \mathrm{mg} / \mathrm{s}$. Xenon was employed as the operation gas for the cathodes. The RF plasma cathode, an anode electrode, and an impedance matching circuit were placed in the chamber, whereas an RF generator, a gas-feed system, and a DC power supply were attached from outside of the chamber. Xenon neutral gas was fed to the vessel as the operation gas via a thermal-sensing mass flow controller. The induction coil was energized at $13.56 \mathrm{MHz}$ by the RF generator via the matching circuit. The incident RF power and the reflected RF power 


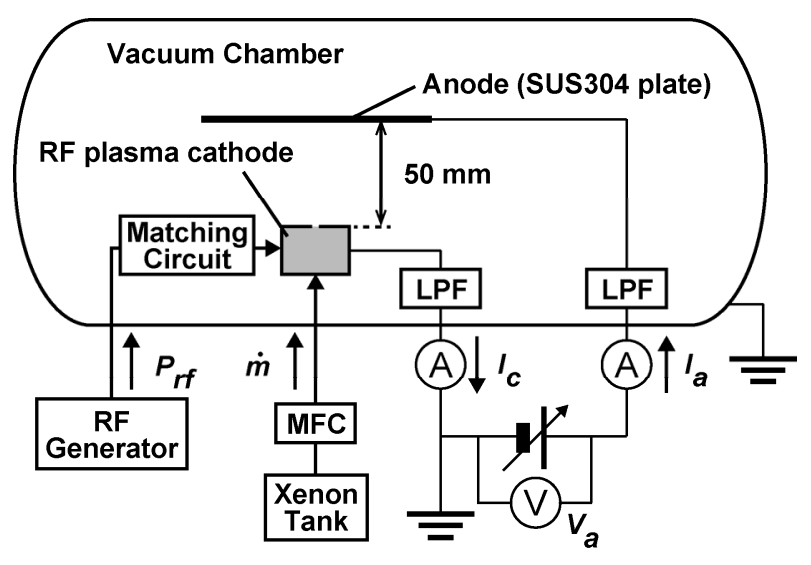

Fig. 3. Experimental setup for performance evaluation of the RF plasma cathodes. "LPF" and "MFC" are a low pass filter and a mass flow controller, respectively.

were measured by a directional coupler in the generator. The matching circuit consists of a series variable capacitor and a parallel variable capacitor. Because the circuit matched the impedance of the generator to that of the $\mathrm{RF} / \mathrm{C}$, there were not reflections of RF power in the experiments. Therefore, in this paper, "RF power" is equal to the net RF power into the cathode.

The plate anode was located downstream of the cathode, and it was made of stainless steel. The distance between the cathode and the anode was $50 \mathrm{~mm}$. The anode was biased positively by the DC power supply to extract electrons from the cathode, while the cathode was connected to the ground potential. When electrons are emitted from the cathode as a result of this potential difference, an electron current is carried to the anode and an ion current is carried to the cathode: these currents are called the anode current and cathode current, respectively. To close the current loop, the anode current is made equal to the cathode current $\left(I_{a}=I_{c}\right)$. The magnitude of the anode current at a constant RF power and xenon mass flow rate indicates the electron emission performance of an electron source. Therefore, to evaluate the electron emission performance, the anode current was measured as a function of the RF power, xenon mass flow rate, and anode voltage.

\section{Results and Discussion}

\subsection{Typical electron emission characteristics of RF plasma cathodes}

Figure 4 shows the anode current for the RF plasma cathodes as a function of the anode voltage at an RF power of $40 \mathrm{~W}$ and a xenon mass flow rate of $0.3 \mathrm{mg} / \mathrm{s}$. The data presented in Fig. 4 show the typical electron-emission characteristics of the RF cathodes. In Fig. 4, the anode current sharply increased as the anode voltage increased, and the anode current remained approximately constant at a higher anode voltage. A bright plasma plume between the orifice and the anode was observed after the anode current sharply increased. From the sharp increase in the anode current and the appearance of the plasma, electrons emitted from the cathode are accelerated by the anode potential to ionize xenon

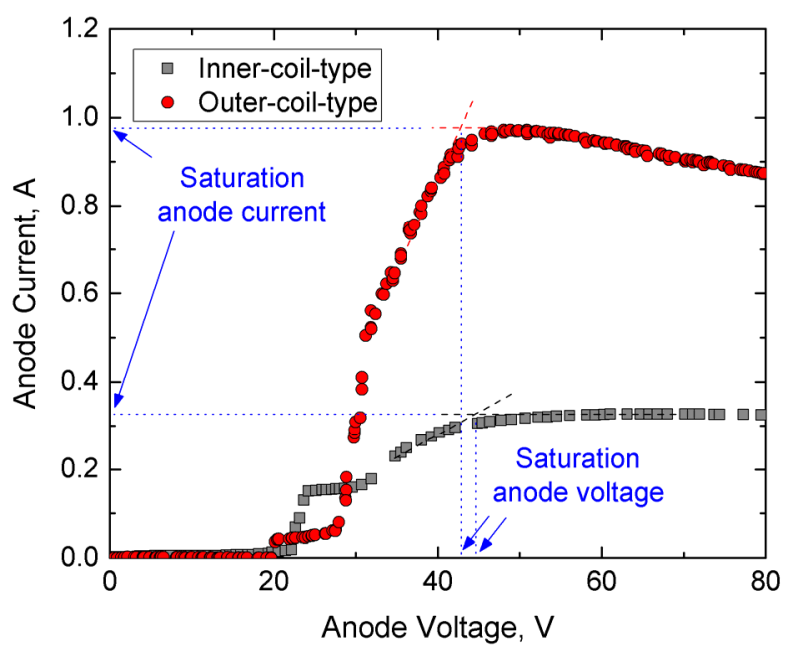

Fig. 4. Anode current for the two RF plasma cathodes as a function of anode voltage at RF power of $40 \mathrm{~W}$ and xenon mass flow rate of $0.3 \mathrm{mg} / \mathrm{s}$.

neutral atoms, and the plasma bridge which reduces the impedance between the cathode and the anode is established. The increase in the anode voltage does not affect the electronemission current from the RF plasma cathode once the plasma bridge was established. In this paper, the anode current saturated at the higher anode voltage is called the "saturation anode current", and the minimum anode voltage to reach the saturation anode current is called the "saturation anode voltage," as shown in Fig. 4. In space applications, it is desirable for electron sources to emit a higher electron current at a lower input power and using lower gas consumption. Therefore, the saturation anode current and the saturation anode voltage are performance indicators for the RF plasma cathode.

\subsection{Outer-coil-type RF plasma cathode}

Figure 5 shows the saturation anode current of the outercoil-type RF plasma cathode as a function of the RF power at various xenon mass flow rates. The data presented in Fig. 5 show that the saturation anode current proportionally increased as the RF power increased. On the other hand, the effect of the xenon mass flow rate on the anode current at a constant RF power is small. Figure 6 shows the saturation anode voltage of the outer-coil-type RF plasma cathode as a function of the RF power at various xenon mass flow rate. The anode saturation voltage increased as the RF power increased. In contrast to the relationship between the saturation anode current and xenon mass flow rate, the saturation anode voltage sharply decreased from 0.1 to $0.3 \mathrm{mg} / \mathrm{s}$, and slowly decreased over $0.3 \mathrm{mg} / \mathrm{s}$, as shown in Fig. 6. An anode current of $3.3 \mathrm{~A}$ was obtained at an RF power of $140 \mathrm{~W}$, a xenon mass flow rate of $0.3 \mathrm{mg} / \mathrm{s}$, and an anode voltage of $58 \mathrm{~V}$. The anode current is sufficiently high to operate a $1-\mathrm{kW}$ class Hall thruster.

To maintain the quasi-neutrality in the RF plasma cathode, the quantity of electrons emitted from the cathode has to be balanced by an equal quantity of ions collected at the ion collector. When ions are collected by the collector, an ion sheath is formed on the collector surface. The ion saturation current in a collision less ion sheath at a low ion temperature 


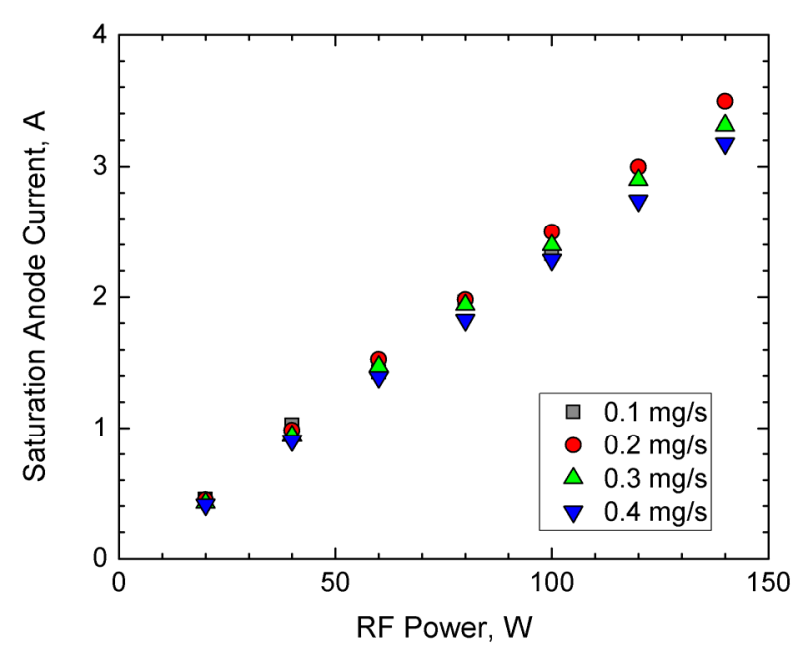

Fig. 5. Saturation anode current of the outer-coil-type RF plasma cathode as a function of RF power at various xenon mass flow rates.

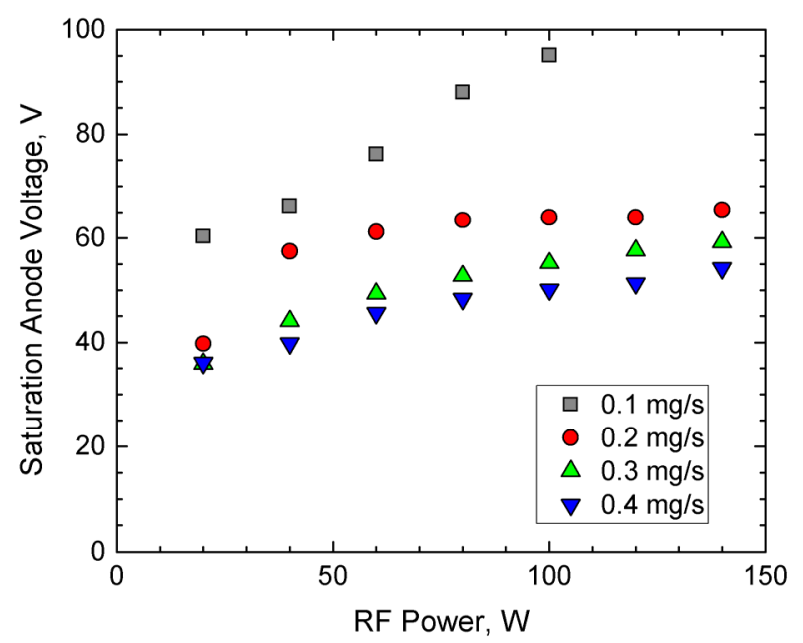

Fig. 6. Saturation anode voltage of the outer-coil-type RF plasma cathode as a function of RF power at various xenon mass flow rates.

is proportional to the ion number density on the sheath edge. The ion number density is typically assumed to be equal to the electron number density in bulk plasma. Sugai ${ }^{27)}$ showed that the ion number density in the ICP with a Faraday shield is proportional to the input RF power. Therefore, we consider that the saturation anode current, which depends on the ion saturation current in the sheath, is proportional to the RF power, as shown in Fig. 5.

\subsection{Inner-coil-type RF plasma cathode}

Figure 7 shows the saturation anode current of the inner-coil-type RF plasma cathode as a function of the RF power at various mass flow rates. As with the electronemission characteristics of the outer-coil-type RF plasma cathode, the saturation anode current increased as the RF power increased. However, there is an inflection point between the saturation anode current and the RF power. Figure 8 shows the saturation anode voltage of the inner-coiltype RF plasma cathode as a function of the RF power at various mass flow rates. The saturation anode voltage increased as the RF power increased and the xenon mass flow

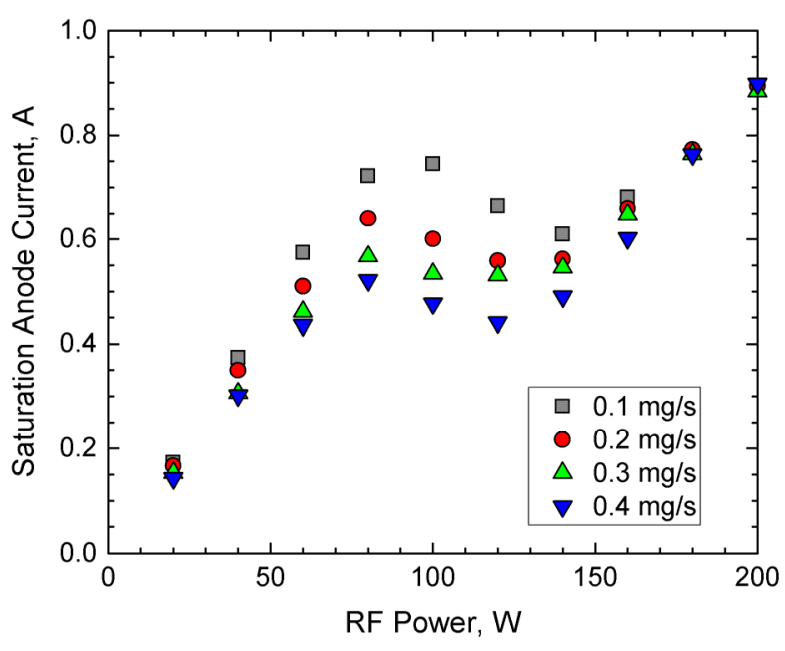

Fig. 7. Saturation anode current of the inner-coil-type RF plasma cathode as a function of RF power at various xenon mass flow rates.

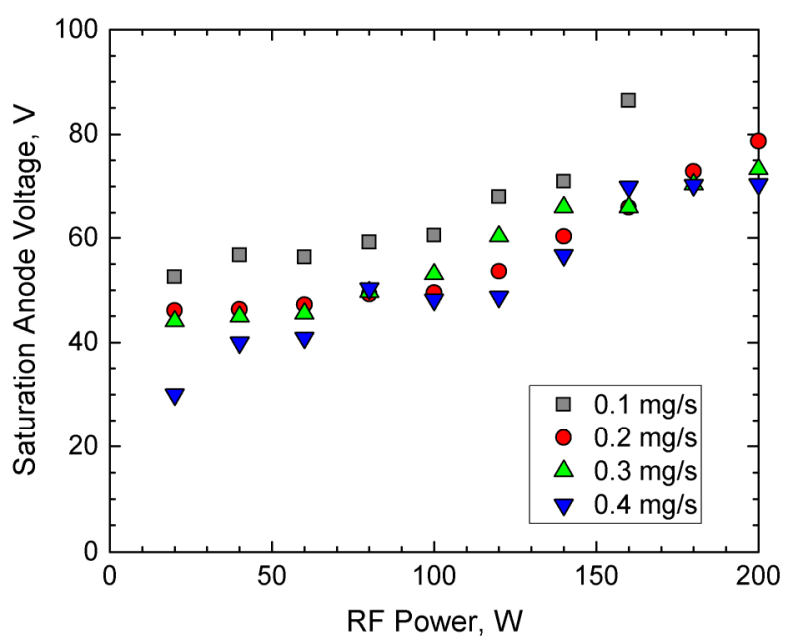

Fig. 8. Saturation anode voltage of the inner-coil-type RF plasma cathode as a function of RF power at various xenon mass flow rates.

rate decreased. The saturation anode current for the outer-coiltype RF plasma cathode is much higher than that for the innercoil-type RF plasma cathode at a constant RF power and xenon mass flow rate. Therefore, we determined that the influence of the coil configuration on the electron-emission characteristics in the RF plasma cathodes is significant.

Figure 9 shows the anode current of the inner-coil-type RF plasma cathode as a function of the anode voltage at an RF power of $200 \mathrm{~W}$ and xenon mass flow rate of $2.0 \mathrm{mg} / \mathrm{s}$. At the high RF power and high xenon mass flow rate, the transition phenomena for an anode current ranging from less than $1 \mathrm{~A}$ to over 12 A was observed as the applied anode voltage increased, as shown in Fig. 9. A high current operation over $12 \mathrm{~A}$ is desirable for use in a high-power Hall thruster operation. However, the transition phenomena and the high current operation were unstable. Thus, in future works, it is necessary to perform additional experimental evaluations to clarify the transition phenomena and the high current operation. 


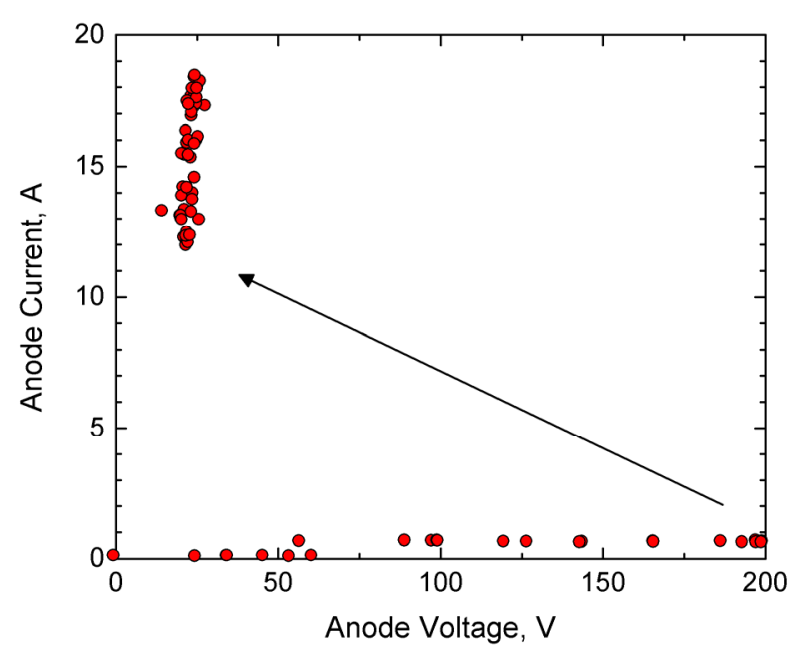

Fig. 9. Anode current of the inner-coil-type RF plasma cathode as a function of anode voltage at RF power of $200 \mathrm{~W}$ and xenon mass flow rate of $2.0 \mathrm{mg} / \mathrm{s}$.

\subsection{Performance comparison of hollow cathode and RF plasma cathodes}

The gas utilization factor and the electron production cost are introduced to evaluate the cathode performance. If the entire xenon fed to the cathode is ionized, $0.1 \mathrm{mg} / \mathrm{s}$ of the xenon fed to the cathode is equivalent to $0.073 \mathrm{~A}$. The gas utilization factor, $U_{e}$, indicates the average number of times that each xenon atom repeats the ionization and recoupling on the cathode surface while the xenon atom remains in the cathode. Thus, it is defined as follows:

$$
U_{e}=\frac{I_{a}[\mathrm{~A}]}{0.073[\mathrm{~A} / \mathrm{mg} / \mathrm{s}] \times \dot{m}_{c}[\mathrm{mg} / \mathrm{s}]}
$$

where $I_{a}$ and $\dot{m}_{c}$ are the anode current and xenon mass flow rate, respectively. The electron production cost, $C_{e}$, is defined as the electron energy that is required for plasma production and electron extraction per $1 \mathrm{~A}$ of electron current as in the following equations.

$$
\begin{gathered}
C_{e}=\frac{I_{a} V_{a}+P_{r f}}{I_{a}} \text { (for RF plasma cathode). } \\
C_{e}=\frac{I_{a} V_{a}+I_{k} V_{k}}{I_{a}} \text { (for hollow cathode). }
\end{gathered}
$$

In Eqs. (2) and (3), $V_{a}, P_{r f}, I_{k}$, and $V_{k}$ are the anode voltage, RF power, keeper current, and keeper voltage, respectively. A higher gas utilization factor and lower electron production cost are desirable for space applications, because the gas and power consumption are limited in space.

Both performance indicators for the RF plasma cathodes and the HCN-252 hollow cathode are shown in Fig. 10. The data for the HCN-252 hollow cathode in Fig. 10 were obtained when the anode current ranged from $2 \mathrm{~A}$ to $10 \mathrm{~A}^{28)}$. Compared to the inner-coil-type RF plasma cathode, the outer-coil-type RF plasma cathode achieved a higher electron-emission performance. The data presented in Fig. 10 show that the gas utilization factor for the outer-coil-type RF plasma cathode is comparable to that for the hollow cathode. On the other hand,

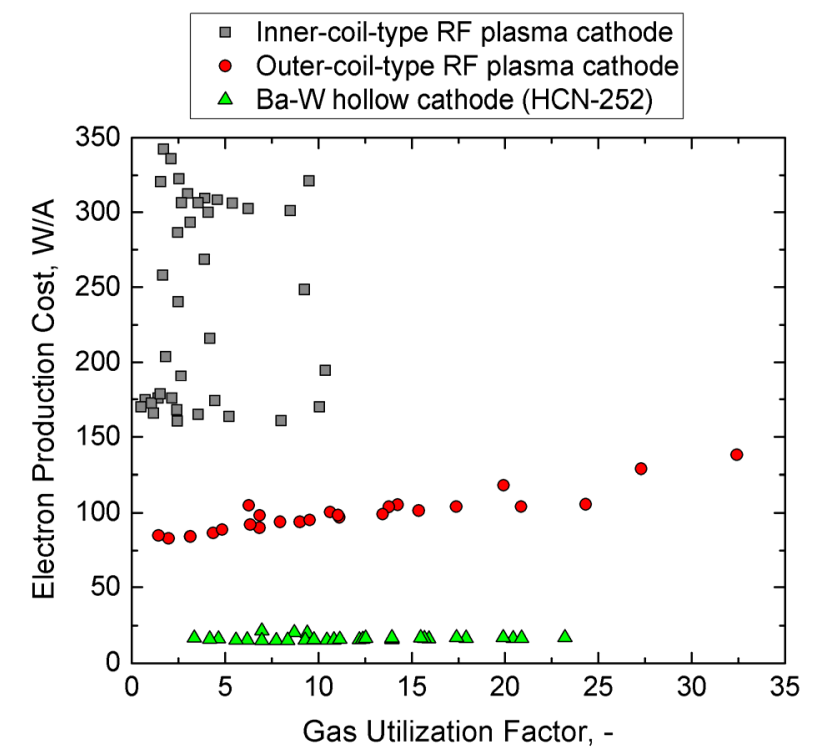

Fig. 10. Electron production cost for the RF plasma cathodes and $\mathrm{HCN}-252$ hollow cathode as a function of gas utilization factor.

the electron production cost for the outer-coil-type RF plasma cathode is four times higher than that for the hollow cathode. This result confirms that for space applications, it is important to improve the power consumption of the RF plasma cathodes.

\section{Conclusion}

To prevent the Hall thrusters from experiencing the shortcomings of the dispenser hollow cathode, we constructed and evaluated an outer-coil-type RF plasma cathode and an inner-coil-type RF plasma cathode. The results can be summarized as follows:

(1) The saturation anode current for the outer-coil-type RF plasma cathode is much higher than that for the inner-coiltype RF plasma cathode at a constant RF power and xenon mass flow rate. Therefore, we determined that the influence of the coil configuration on the electronemission characteristics in the RF plasma cathodes is significant.

(2) Compared to the inner-coil-type RF plasma cathode, the outer-coil-type RF plasma cathode achieved higher electron-emission performance. In the outer-coil-type RF plasma cathode, an anode current of $3.3 \mathrm{~A}$ was obtained at an RF power of $140 \mathrm{~W}$, a xenon mass flow rate of 0.3 $\mathrm{mg} / \mathrm{s}$, and an anode voltage of $58 \mathrm{~V}$. The anode current is sufficiently high to operate a 1-kW class Hall thruster.

(3) For a high RF power and high xenon mass flow rate during the operation of the inner-coil-type RF plasma cathode, the transition phenomena for an anode current that ranges from less than $1 \mathrm{~A}$ to over $12 \mathrm{~A}$ was observed as the applied anode voltage increased. Because the transition phenomena and the high current operation were unstable, additional experimental evaluations are required to clarify the transition phenomena and the high current operation.

(4) The gas utilization factor for the outer-coil-type RF plasma cathode is comparable to that for a conventional dispenser hollow cathode. On the other hand, the electron production 
cost for the outer-coil-type RF plasma cathode is four times higher than that for the hollow cathode. This result verifies that improvements in the power consumption are important for the application of RF plasma cathodes to Hall thrusters.

\section{Acknowledgments}

The authors would like to thank Dr. K. Kuriki (Professor Emeritus, Institute of Space and Astronautical Science) and Dr. I. Funaki (Associate Professor, Institute of Space and Astronautical Science) for helpful discussions and technical supports. This study was supported by a Grant-in-Aid for Young Scientists (B), No. 25820415, sponsored by the Japan Society for the Promotion of Science.

\section{References}

1) D. M. Goebel, and I. Katz: Fundamentals of Electric Propulsion Ion and Hall Thrusters, JPL Space Science and Technology series, Wiley \& Sons, 2008, Chap. 9.

2) K. Grys, A. Mathers, B. Welander and V. Khayms: Demonstration of 10,400 Hours of Operation on a $4.5 \mathrm{~kW}$ Qualification Model Hall Thruster, AIAA Paper 2010-6698, 2010.

3) V. Kim, G. Popov, B. Arkhipov, V. Murashko, O. Gorshkov, A. Koroteyev, V. Garkusha, A. Semenkin and S. Tverdokhlebov: Electric Propulsion Activity in Russia, 27th International Electric Propulsion Conference, IEPC-2001-005, 2001.

4) S. R. Koppel, F. Marchandise, D. Estublier and L. Joliver: The SMART-1 Electric Propulsion Subsystem in Flight Experience, AIAA Paper 2004-3435, 2004.

5) A. Mathers, K. Grys and J. Paisley: Performance Variation in BPT4000 Hall Thrusters, 31st International Electric Propulsion Conference, IEPC-2009-144, 2009.

6) J. L. Cronin: Modern dispenser cathode, IEE Proceedings Part I: Solid-State and Electron Devices, 128 (1981), pp. 19-31.

7) A. H. W. Beck: High-current-density Thermionic Emitters: A Survey, IEE Proceedings Part B: Electronic and Communication Engineering, 106 (1959), pp. 372-390.

8) J. L. Cronin: Practical aspects of modern dispenser cathodes, Microwave Journal, 22 (1979), pp. 57-62.

9) I. G. Mikellides and I. Katz: Wear Mechanisms in Electron Sources for Ion Propulsion, 1: Neutralizer Hollow Cathode, Journal of Propulsion and Power, 24 (2008), pp. 855-865.

10) I. G. Mikellides, I. Katz, D. M. Goebel, K. K. Jameson and J. E. Polk: Wear Mechanisms in Electron Sources for Ion Propulsion, 2: Discharge Hollow Cathode, Journal of Propulsion and Power, 24 (2008), pp. 866-879.

11) J. E. Polk, I. G. Mikellides, I. Katz and A. M. Capece: Tungsten and barium transport in the internal plasma of hollow cathode, Journal of Applied Physics, 105 (2009), 113301.
12) M. Coletti and S. B. Gabriel: Barium Oxide Depletion from Hollow- Cathode Inserts: Modeling and Comparison with Experiments, Journal of Propulsion and Power, 26 (2010), pp. 364-369.

13) Y. Ohkawa, T. Higuchi, Y. Hayakawa, K. Miyazaki amd H. Nagano: Observation and Analysis of Graphite Hollow Cathode after 45,000-Hour Life Test, 33rd International Electric Propulsion Conference, IEPC-2013-364, 2013.

14) D. M. Goebel and R. M. Watkins: Compact lanthanum hexaboride hollow cathode, Review of Scientific Instruments, 81 (2010), 083504.

15) D. J. Warner, R. D. Branam and W. A. Hargus Jr.: Ignition and Plume Characteristics of Low-Current Cerium and Lanthanum Hexaboride Hollow Cathodes, Journal of Propulsion and Power, 26 (2010), pp. 130-134.

16) E. M. Oks: Physics and technique of plasma electron sources, Plasma Source Science and Technology, 1 (1992), pp. 249-255.

17) I. Funaki and H. Kuninaka: Overdense Plasma Production in a Low-power Microwave Discharge Electron Source, Japanese Journal of Applied Physics, 40 (2001), pp. 2495-2500.

18) B. R. Weatherford, J.E. Foster amd H. Kamhawi: Electron current extraction from a permanent magnet waveguide plasma cathode, Review of Scientific Instruments, 82 (2011), 093507.

19) L. Liard, Y. Zhu, G. J. M. Hagelaar and J. -P. Boeuf: Fluid simulation of a microwave plasma cathode, 33rd International Electric Propulsion Conference, IEPC-2013-396, 2013.

20) St. Weis, K. - H. Schartner, H. Loeb and D. Feili: Development of a capacitively coupled insert-free RF-neutralizer, 29th International Electric Propulsion Conference, IEPC-2005-086, 2005.

21) F. Scholze, M. Tartz and H. Neumann: Inductive coupled radio frequency plasma bridge neutralizer, Review of Scientific Instruments, 79 (2008), $02 \mathrm{~B} 724$.

22) Y. Raitses, J. K. Hendryx and N. J. Fisch: A Parametric Study of Electron Extraction from a Low Frequency Inductively Coupled RF-Plasma Source, 31st International Electric Propulsion Conference, IEPC- 2009-024, 2009.

23) H. Watanabe, T. Okuma, J. Aoyagi and H. Takegahara: Research and Development on Inductively Coupled Plasma Cathode for Ion Engines, 31st International Electric Propulsion Conference, IEPC-2009-027, 2009.

24) B. Longmier and N. Hershkowitz: Improved operation of the nonambipolar electron source, Review of Scientific Instruments, 79 (2008), 093506.

25) H. Kuninaka, K. Nishiyama, Y. Shimizu, I. Funaki, H. Koizumi, S. Hosoda and D. Nakata: Hayabusa Asteroid Explorer Powered by Ion Engines on the way to Earth, 31st International Electric Propulsion Conference, IEPC-2009-267, 2009.

26) M. A. Lieberman and A. J. Lichtenberg: Principles of Plasma Discharges and Materials Processing, 2nd ed., John Wiley \& Sons, 2005, Chap. 12.

27) H. Sugai, K. Nakamura and K. Suzuki: Electrostatic Coupling of Antenna and the Shielding Effect in Inductive RF Plasma, Japanese Journal of Applied Physics, 33 (1994), pp. 2189-2193.

28) S. Sakai, T. Katayama, J. Aoyagi and H. Takegahara: Discharge Modes and Characteristics of Hollow Cathode, 30th International Electric Propulsion Conference, IEPC-2007-215, 2007. 